\title{
ВЛИЯНИЕ ИНФОРМАЦИОННОЙ СРЕДЫ НА РАЗВИТИЕ РАКЕТНО-КОСМИЧЕСКОЙ ОТРАСЛИ
}

\author{
(c) 2021 Конников Евгений Александрович \\ кандидат экономических наук, доцент, Высшая инженерно-экономическая школа \\ Санкт-Петербургский политехнический университет Петра Великого, Россия, Санкт-Петербург \\ E-mail: konnikov.evgeniy@gmail.com \\ (C) 2021 Крыжко Дарья Александровна \\ Высшая инженерно-экономическая школа \\ Санкт-Петербургский политехнический университет Петра Великого, Россия, Санкт-Петербург \\ E-mail: kryzhko_da@spbstu.ru \\ (c) 2021 Шадров Кирилл Сергеевич \\ Высшая инженерно-экономическая школа \\ Санкт-Петербургский политехнический университет Петра Великого, Россия, Санкт-Петербург \\ E-mail: micekillor9@mail.ru
}

Одной из наиболее капиталоемких и вариативных с точки зрения ценности получаемого результата является ракетно-космическая отрасль, несмотря на многомерное развитие данной отрасли на протяжении последних десятилетий. При этом интенсивное развитие данной отрасли в последние годы обусловлено появлением первых коммерческих предприятий. Таким образом, несмотря на значимую, в первую очередь с экономической точки зрения, вариативность результатов, данная отрасль привлекает значительный объём инвестиций. В рамках данного исследования рассматривается данный инвестиционный поток - как центральный фактор результативности. Ключевой гипотезой данного исследования, является утверждение о потенциальной зависимости данного фактора от состояния информационной среды, вследствие чего целью исследования является количественная оценка данной зависимости.

Ключевые слова: информационная среда, ракетно-космическая отрасль, объем финансирования отрасли, исследования космоса.

За последние 20 лет область исследования космоса претерпела огромное количество существенных изменений, основным среди которых является открытие данной отрасли для деятельности частных компаний, а значит и для открытой конкуренции, которая приводит к форсированному развитию. Буквально в 2019 году была запущена первая полностью частная ракета для стыковки с MKC, Crew Dragon, а значит частные компании добрались до уровня государственных программ и теперь устремлены к улучшению и дальнейшему развитию космической отрасли. Подобные программы играют значимую роль в жизни всего общества, глобально влияя на политику и экономику всех развитых и развивающихся стран, объединяя или же наоборот разделяя мир на противоборствующие лагеря. Именно поэтому сейчас, у предполагаемых истоков новой вехи освоения космоса полезно будет изучить как развитие космических технологий и популяризация значимых событий сказывается на действительных показателях фактических запусков. Кроме того, успех коммерческих космических компаний, таких как SpaceX до сих пор является горячо обсуждаемой темой множества дискуссий, как в кругах учёных и инвесторов, так и среди обывателей.

Одним из передовых изобретений человечества, считается изобретение Интернета. На уровне обывателя он может показаться всего лишь местом для поиска информации, передачи информации и общения на расстоянии, но если взглянуть на него с другой стороны, то Интернет предстаёт силой, способной менять не только жизни конкретных людей, но и целых сообществ. Целью данной работы является анализ и выявление стран-лидеров, обладающих достаточно прочной экономикой, позволяющей инвестировать в проекты, помогающие аэрокосмической отрасли, а также нахождение того уровня, 
на который фокус промышленности сдвигается в сторону освоения космоса, а значит выявление градуса влияния общественного интереса к теме развития аэрокосмических программ, на фактические показатели количества запусков стран-лидеров, а также и влиянии реакции общественности на эту деятельность на общий объем финансирования, выделяемого странами на отрасль космической промышленности.

Для решения поставленной цели исследования необходимо вывести потенциальные уравнения, а затем их обосновать благодаря сбору теоретической информации и по результатам регрессионного анализа. Первоначальный вариант концептуальной модели представлен на рисунке 1.

Стоит учитывать, что для того, чтобы определить итоговый вариант модели необходимо провести регрессионный анализ и сбор дополнительных данных. Важно определить ключевые характеристики распределения данных в рамках концептуальной модели, среди которых:

- данные имеют панельную структуру и ограничения, связанные с недостаточным объемом необходимой информации в официальных источниках;

- при использовании кода могут возникнуть ошибки с высокой степенью апроксимации ввиду искусственных ограничений данного инструмента

- показатель допустимой ошибки равен 0,05 для каждой из рассмотренных в расчётной части моделей;

- показатель «Присутствие в новостях» был получен с использованием кода на основе Python.

Оптимальным источником первичной информации в данном случае выступает платформа агрегации новостей от google, что в первую очередь обусловлено эффективными алгоритмами google, позволяющими идентифицировать наиболее широко распространённые информа-

Квантификаторы состояния информационной среды сферы космических исследований

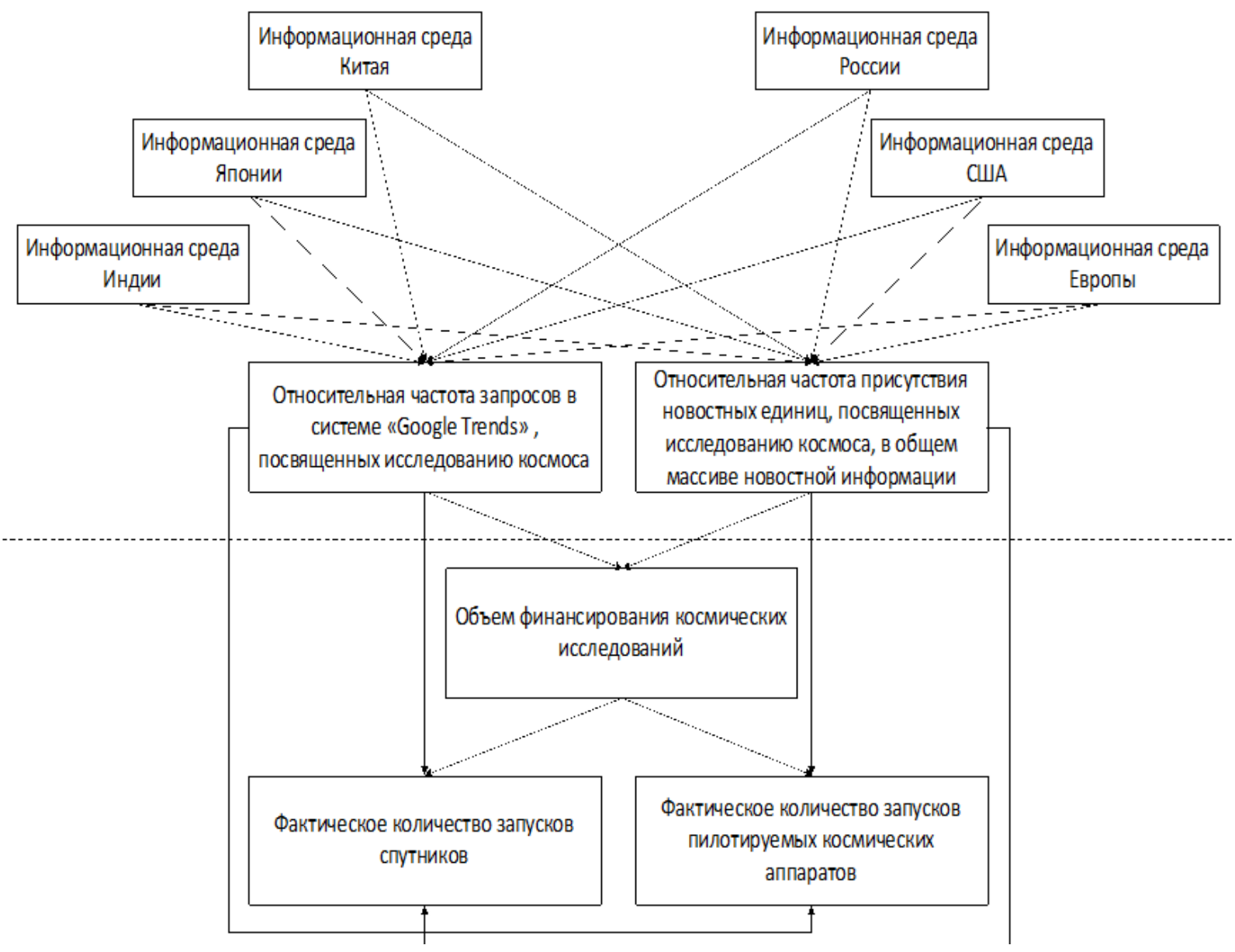

Рисунок 1. Концептуальная модель исследования 
ционные посылы. Для целей автоматизированного парсинга данной информации может быть использован язык программирования Python 3 и библиотека py Google News. Инструменты данной библиотеки позволяют определить наиболее широко распространённые новостные заголовки в рамках заданного периода времени и на заданном языке. Перечень показателей, используемых при анализе представлен в таблице 1.

Выводя гипотезы и анализируя собранные данные, мы можем составить ряд концептуальных уравнений, утверждающих ту или иную связь между показателями. Далее представлен набор уравнений, изучающих все логически обоснованные связи между данными показателями:

$$
\left\{\begin{array}{c}
y=\mathrm{a}+b 1 * x 1+b 2 * x 2+b 3 * x 3 \\
x 1=a+b 2 * x 2+b 3 * x 3 \\
y=a+b 2 * x 2+b 3 * x 3 \\
y=\mathrm{a}+b 1 * x 1
\end{array}\right.
$$

Первое уравнение характеризует влияние объемов финансирования на фактическое количество запусков. Второе уравнение связано с оценкой влияния общественного интереса на объемы финансирования космических программ. Третье - позволяет провести анализ влияния общественного интереса на фактическое количество запусков в разных странах. Четвертое уравнение связано с оценкой влияния всех экзогенных факторов на фактическое количество запусков в разных странах. Критерии, на основании на которых будет производиться исследование: p-level, коэффициент детерминации $\mathrm{R}^{2}, \mathrm{~F}$-критерий Фишера, ошибка аппроксимации А, относительный коэффициент эластичности Э, этот ряд показателей регрессионного анализа был выбран в силу наглядности и удобности использования каждого из данных показателей. Рассмотрим последовательно каждое из уравнений.
Уравнение первое - влияние объемов финансирования на фактическое количество запусков. Регрессионный анализ не выявил необходимости в оптимизации модели, показатели не выходят за пределы нормы. Вид окончательного уравнения:

$$
y=11,35+0,30 x 1
$$

Где:

У - фактическое количество запусков;

$\mathrm{X} 1$ - объем финансирования космических программ.

Коэффициент b1 имеет положительное значение, так как количество запусков напрямую зависит от объемов финансовых инвестиций, что абсолютно логично, ошибка аппроксимации высокая ввиду малого количества наблюдений и панельного расположения статистических данных. Значимость F составляет примерно 0,00449, что находится в пределах нормы, а значит модели можно доверять. $\mathrm{R}^{2}$ равен 0,254 , что значит, что построенная модель объясняет 25 процентов описанной дисперсии, из чего можем сделать вывод о том, что на исследуемый показатель влияет гораздо большее количество факторов, чем можно было изучить. P-level показателя финансирования равен 0,00449 , что значит, что изменение значений показателя финансирования объективно отражает изменение показателя У.

Рассмотрим расположение теоретических значений У в соответствии с фактическими показателями в пределах границ, выведенных с использованием ошибки аппроксимации (рисунок 2).

В соответствии с данными, представленными на рисунке 2 структурные разрывы отсутствуют, но существуют определенные выбросы, т.к фактическое количество запусков зависит от большого количества факторов, таких как, например, большая политика, примером можно

Таблица 1. Сводная таблица показателей, используемых при анализе

\begin{tabular}{|c|l|c|c|c|}
\hline № & \multicolumn{1}{|c|}{ Наименование показателей } & $\begin{array}{c}\text { Условное } \\
\text { обозначение }\end{array}$ & $\begin{array}{c}\text { Единицы } \\
\text { измерения }\end{array}$ & Тип \\
\hline 1 & Количество запусков спутников & - & Шт. & Эндогенный \\
\hline 2 & Количество запусков пилотируемых аппаратов & - & Шт. & Эндогенный \\
\hline 3 & Итого запусков & У (№ 1+№ 2) & Шт. & Эндогенный \\
\hline 4 & Объем финансирования & $\mathrm{X} 1$ & $\begin{array}{c}\text { Млрд. долла- } \\
\text { ров }\end{array}$ & $\begin{array}{c}\text { Эндогенно- } \\
\text { Экзогенный }\end{array}$ \\
\hline 5 & Запросы в google trends & $\mathrm{X} 2$ & Ед. & Экзогенный \\
\hline 6 & Присутствие в новостях & $\mathrm{X} 3$ & Ед. & Экзогенный \\
\hline
\end{tabular}




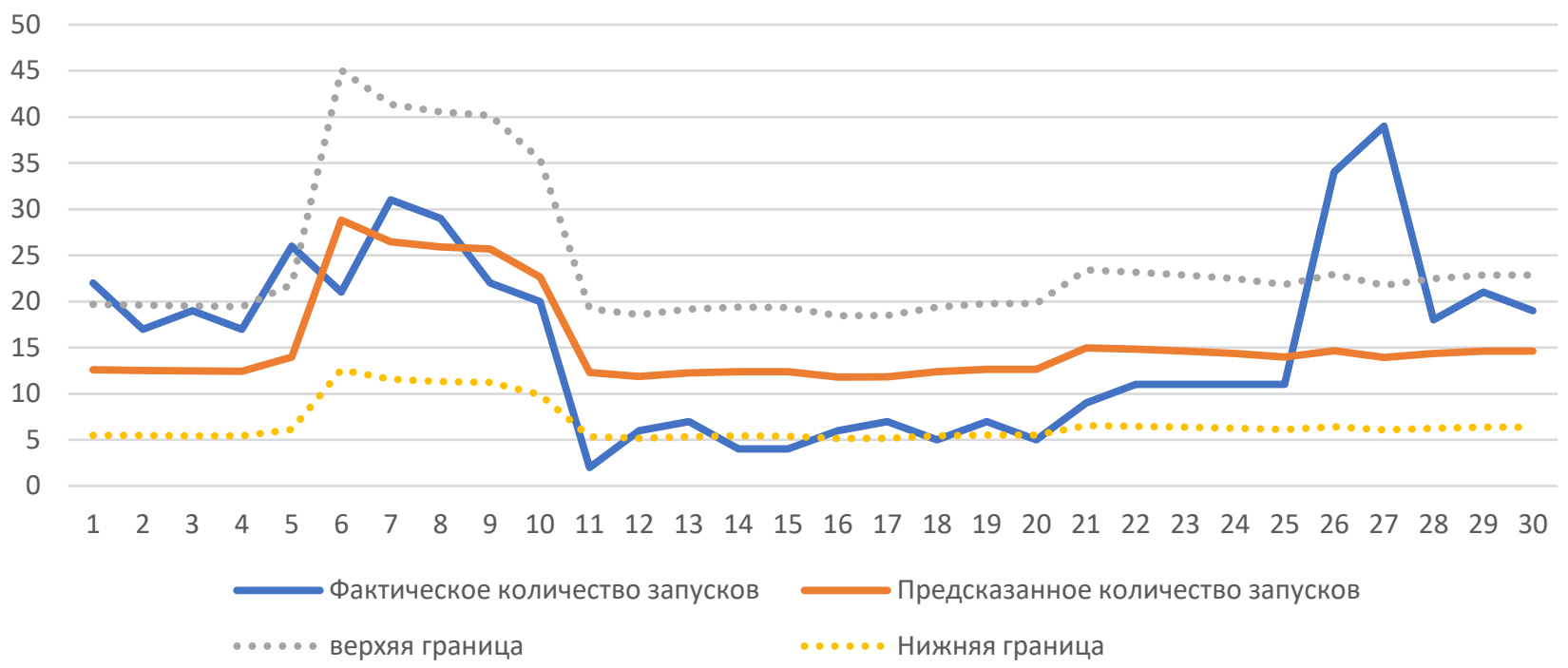

Рисунок 2. График сопоставления фактических и теоретических значений У для первого уравнения

назвать Китайскую космическую программу на 2017-2019 год, когда было значительно увеличено количество запусков за несколько лет в два и более раза. График остатков для данного уравнения представлен на рисунке 3.

Расположение остатков представляет собой панельную структуру, что является характерной особенностью космической отрасли, так каждой отдельно рассматриваемой стране требуется свой объем финансовых средств, расходуемых на запуски. Стоит учитывать, что данная характеристика обусловлена сложившимися уникальными целями, стандартами и условиями производимых запусков.

Параметр эластичности данного уравнения позволяет определить степень связи между финансированием и фактическим количеством запусков (рисунок 4).

Таким образом, увеличение расходов на финансирование космических программ приведет к фактическому увеличению количества запусков в промежутке от 0,06 процентов и до 0,46 процентов. На этом анализ первого уравнения можно считать завершённым, перейдём к анализу следующих уравнений, используя тот же инструментарий оценки.

Уравнение второе - оценка влияния общественного интереса на объемы финансирования космических программ. Вид окончательного уравнения:

$$
x 1=-18,95+0,0024 \times 2+0,0045 \times 3
$$

Где:

$\mathrm{X} 1$ - объем финансирования космических программ;

X2 - количество запросов в google trends, oxватывающих интересующую нас тематику;

X3 - количество упоминаний интересующей нас тематики в новостном фоне стран.

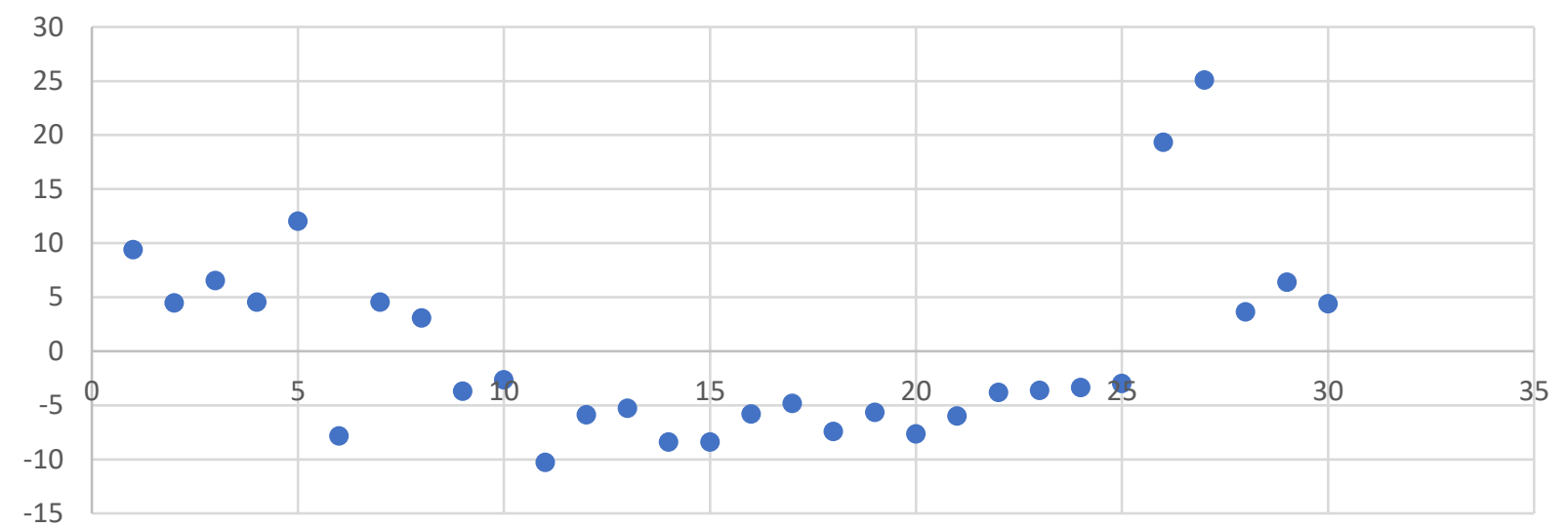

Рисунок 3. График остатков для первого уравнения 


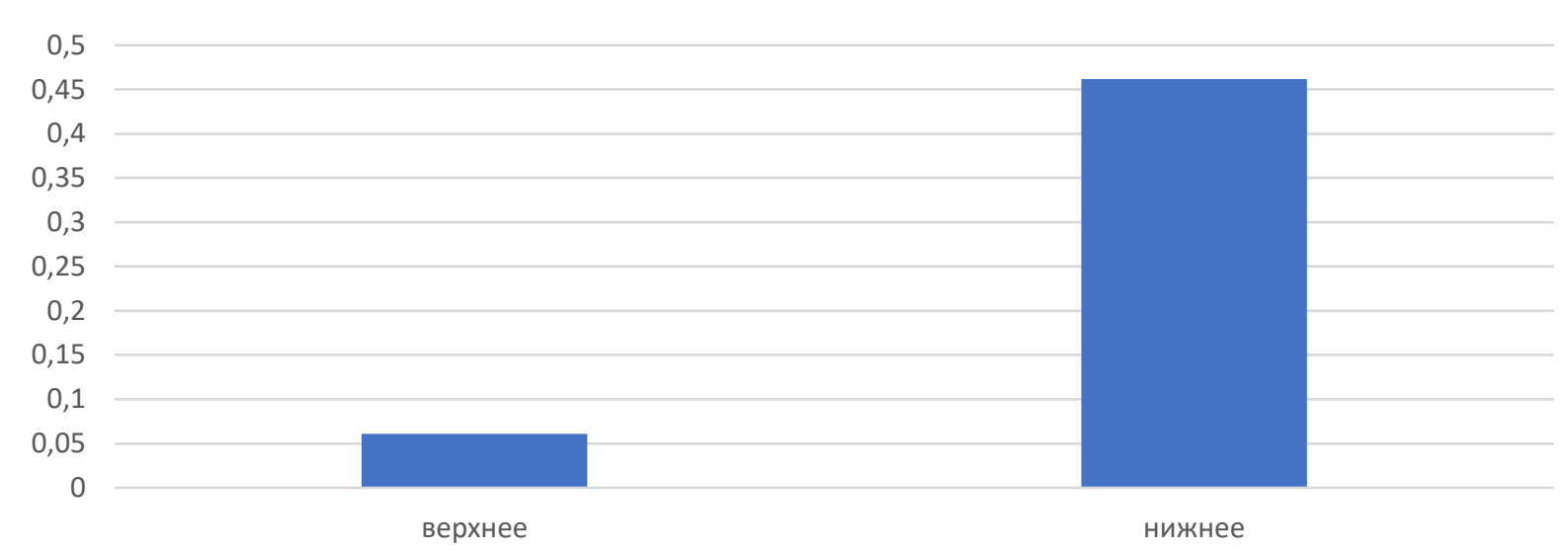

Рисунок 4. Гистограмма нижней и верхней границы доверительного интервала коэффициентов эластичности для х1

Проанализировав данные регрессионного анализа по этим показателям, можно утверждать, что связь существует и объясняется 33 процента описанной дисперсии по значению $\mathrm{R}^{2}$, модели можно доверять в соответствии со значимостью F. Однако в случае ошибки аппроксимации заметно превышение допустимых значений, а следовательно дальнейшее рассмотрение модели нецелесообразно. Следует отметить, что данным уравнением подтверждается наличие связи между общественным интересом к тематике исследования космоса и объемами финансирования космических программ, но либо эта связь настолько незначима, что не позволяет модели исправно функционировать, либо предоставленные данные ввиду своей недостаточности не позволяют точно выверить и измерить данную связь.

Уравнение третье - оценка влияния общественного интереса на фактическое количество запусков в разных странах. Регрессионный анализ не выявил необходимости в оптимизации модели, показатели не выходят за пределы нормы. Вид окончательного уравнения:

$$
y=1,88-0,0014 x 2+0,0065 x 3
$$

Где:

У - фактическое количество запусков;

X2 - количество запросов в google trends, oxватывающих интересующую нас тематику;

X3 - количество упоминаний интересующей нас тематики в новостном фоне стран.

Коэффициент b1 отрицательный, в то время как коэффициент b2 - положительный, отрицательное значение первого коэффициента может быть связано с обратным влиянием его на исследуемый показатель, но более вероятно возникновение ситуации, при которой влияние количества запросов и интереса общества к космической тематике имеет накопительный, долгоиграющий эффект. Высокая ошибка аппроксимации (почти 50 процентов) объясняется относительно малым количеством наблюдений, влиянием сторонних факторов, а также несовершенностью статистических данных. По значению $\mathrm{R}$ квадрата в 0,4373 можно сказать, что модель объясняет почти 44 процента описанной дисперсии. Показатели P-level в пределах допустимой нормы у каждого из двух экзогенных показателей, что значит, что можно отслеживать их объективное влияние на исследуемый показатель количества запусков. Значимость F модели также находится в пределах допустимой нормы не приближаясь к опасным для достоверности границам. Это уравнение доказывает наличие связи между общественным интересом к тематике освоения космоса и ростом фактического показателя запусков космических аппаратов.

Далее необходимо сопоставить теоретические значения, полученные в ходе регрессионного анализа и фактические данные о запусках (рисунок 5).

Структурных разрывов на графике не наблюдается, выходов фактического значения за границы несколько, каждый из них объясняется влиянием ряда сторонних факторов, которые в рамках данной статьи не рассматриваются. Одним из примеров таких показателей может служить деятельность частных американских компаний в отрасли создания новых спутниковых сетей Starlink, первые запуски для которой произошли в 2018 году, что объясняет один из выбросов, присутствующих на данном графике. Следует рассмотреть график расположения остатков в этом уравнении (рисунок 6). 


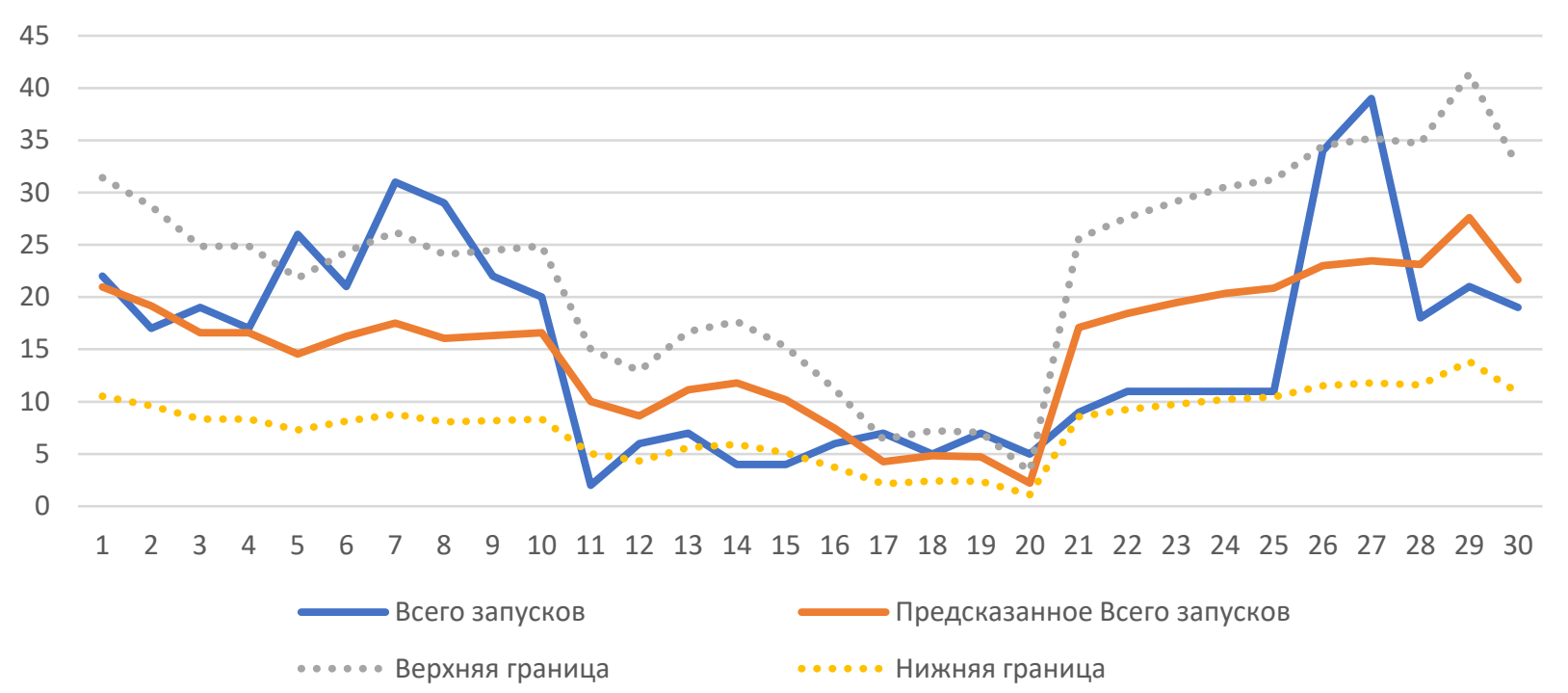

Рисунок 5. График сопоставления фактических и теоретических значений У

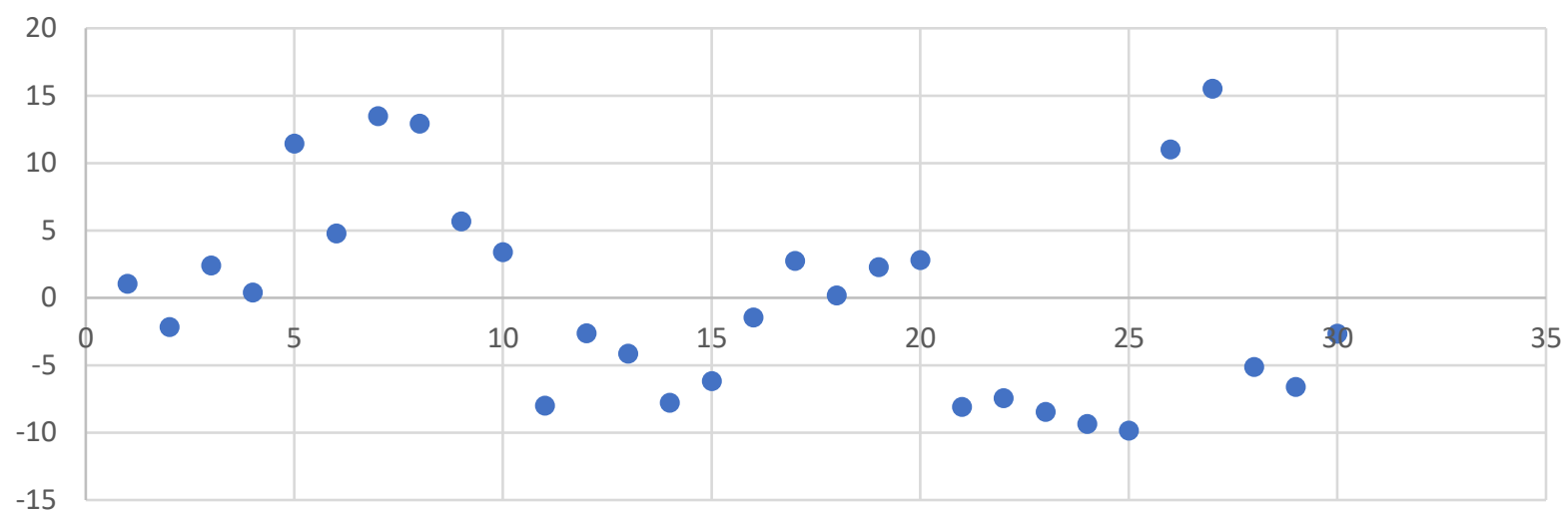

Рисунок 6. График остатков для третьего уравнения

На графике остатков можно в очередной раз отметить нормальное случайное распределение, единственные тренды - это причастность рядов данных к конкретным странам, что отлично демонстрируется на данном графике. Это вызвано тем, что для анализа используются панельные данные, расположенные в специфическом порядке. Мультиколлинеарности между экзогенными показателями не прослеживается, так как значение их VIF меньше критической точки. Далее рассмотрим значения коэффициентов эластичности для этого уравнения (рисунок 7).

Здесь можно отследить, что интерес общества к космической тематике разобщен, т.е изменение одного показателя может обратно пропорционально повлиять на изменение другого, что указывает нам на то, что связь между интересом общества и количеством запусков присутствует, но её сила невелика. Перейдём к рассмотрению последнего уравнения в модели анализирующего влияние всех исследуемых показателей.

Уравнение четвертое - оценка влияния всех экзогенных факторов на фактическое количество запусков в разных странах. Вид окончательного уравнения:

$$
y=7,92+0,32 \times 1-0,0021 \times 2+0,005 \times 3
$$

Где:

у - фактическое количество запусков;

$\mathrm{x} 1$ - объем финансирования космических программ;

х2 - количество запросов в google trends, oxватывающих интересующую нас тематику;

x3 - количество упоминаний интересующей нас тематики в новостном фоне стран.

Коэффициенты b в данном уравнении, так- 


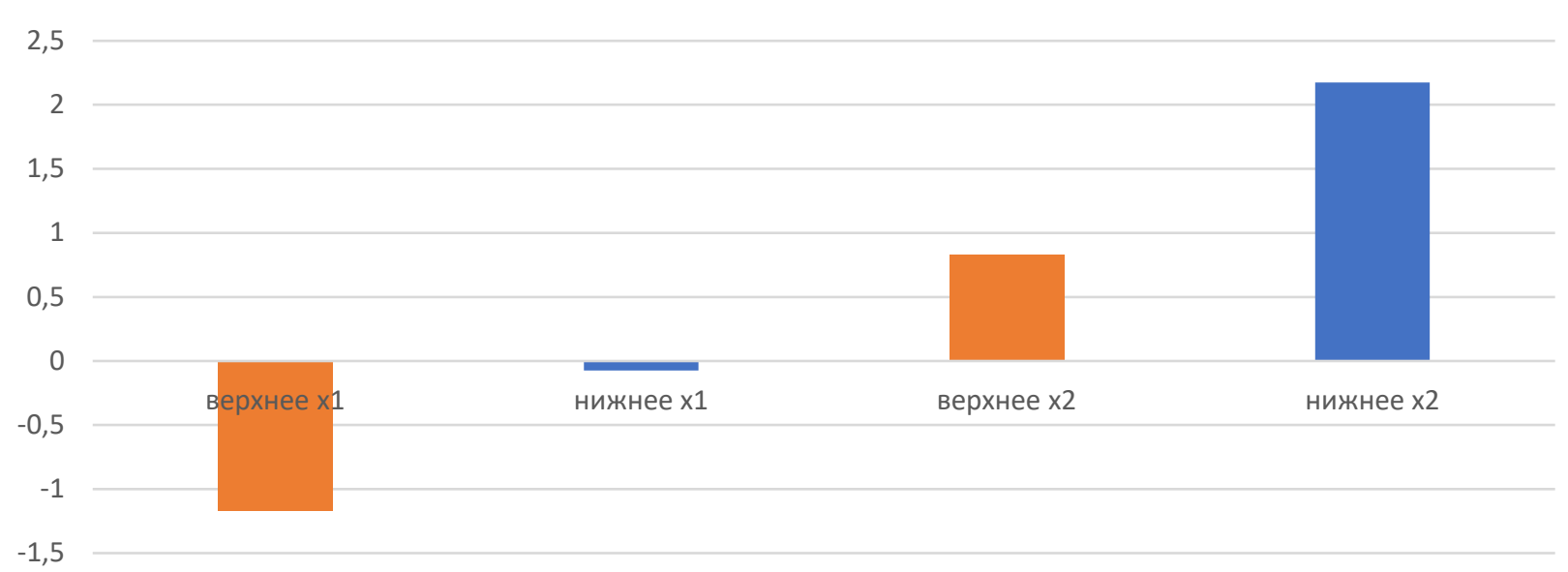

Рисунок 7. Гистограмма нижней и верхней границы доверительного интервала коэффициентов эластичности для х2 и х3

же, как и в предыдущем, имеют как положительные значения, так и отрицательные, что говорит о прямом влиянии финансирования на количество запусков, а также позволяет нам судить о накопительном эффекте влияния интереса общества. Финальное уравнение подвержено меньшей ошибке аппроксимации, чем предыдущие, но она всё ещё составляет около 42 процентов из-за малого количества наблюдений и несовершенности статистических данных ввиду их закрытости и редкости. Значимость $\mathrm{F}$ находится в пределах допустимого и не подходит близко к границе этих пределов, что позволяет нам говорить о достоверности этой модели. Значения P-level каждого из экзогенных показателей находятся в допустимых пределах, что позволяет судить о достоверности их влияния. Значение $\mathrm{R}$ квадрата составляет 0,62506, что говорит о том, что влияние показателей объясняет 62 процента описанной дисперсии. Это хорошее значение, но не стоит забывать о влиянии ряда внешних факторов, влияние которых мы не рассматриваем. В этом уравнении наиболее заметно, что финансирование объясняет наибольшую долю описанной дисперсии, и в сравнении с этим влияние общественного интереса предстаёт в виде едва заметной связи. Сопоставим фактические и теоретические значения У по четвертому уравнению (рисунок 8).

Здесь все закономерности, проявляющиеся на предыдущих графиках, объединяются и сглаживают друг друга, и на выходе получается график без структурных разрывов, указывающий на несколько выбросов, объясняемых влиянием внешних факторов, таких как влияние большой политики, обстоятельств, связанных с новыми проектами, и прочими неизвестными величинами. График остатков для последнего уравнения представлен на рисунке 9.

График остатков не позволяет определить

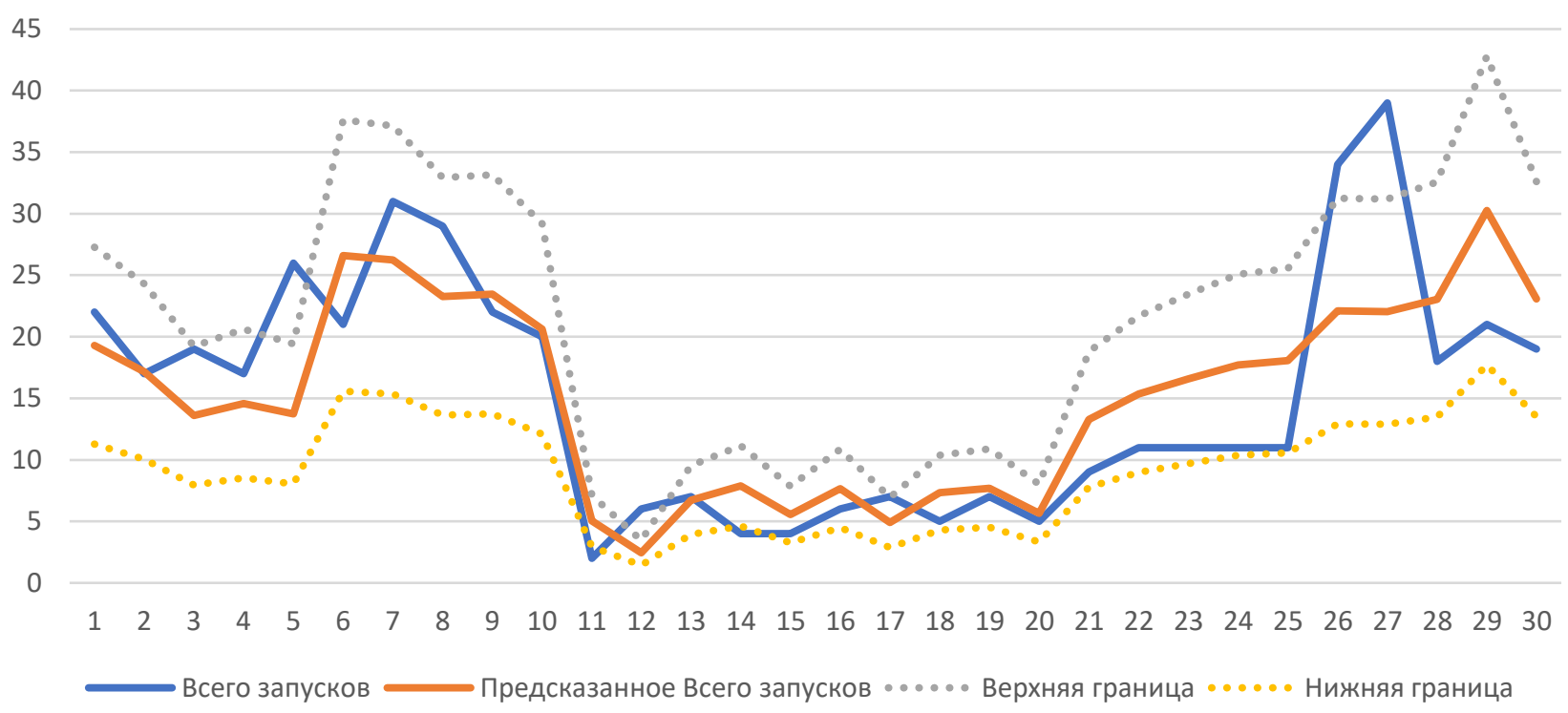

Рисунок 8. График сопоставления фактических и теоретических значений У 


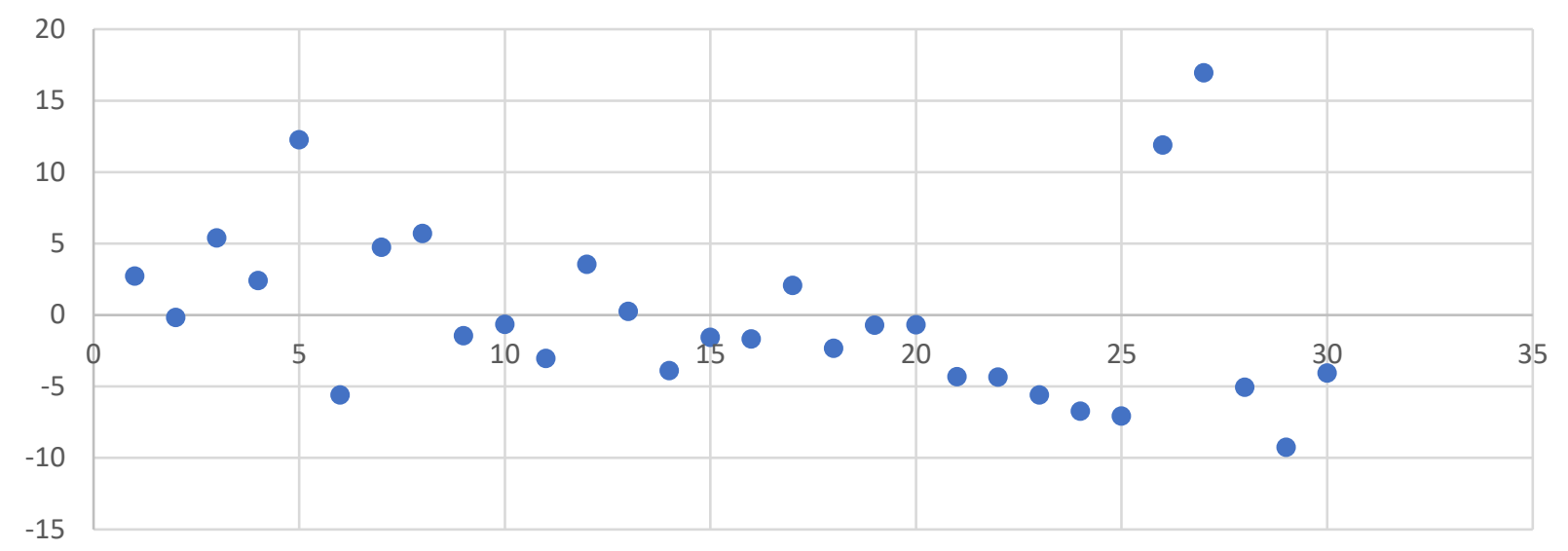

Рисунок 9. График остатков для четвертого уравнения

тренды и гетероскедастичность, так как данные расположены в панельном виде, а остатки распределены нормально, независимо. Мультиколлинеарность отсутствует. Рассмотрим коэффициенты эластичности для каждого из показателей вместе с их верхней и нижней границей для того, чтобы по разрыву определить, каким из показателей можно наиболее эффективно управлять моделью (рисунок 10).

При изменении х1 на 1 процент, остальные факторы изменяются в промежутке от 0,12 процентов до 0,43 процентов, а при увеличении икса они увеличиваются, в этом факторе разрыв минимален. Данный факт указывает на то, что именно изменяя финансирование появляется возможность наиболее эффективного управления изменениями в исследуемом показателе. Влияние следующих показателей сложно предугадать, так как интерес общества даёт большие разрывы между верхней и нижней границей доверительного интервала коэффициентов эластичности. Таким образом, можно сделать вывод о том, что подогревая интерес общества к кос- мической тематике, можно как положительно повлиять на фактическое положение дел в индустрии, так и незначительно ухудшить реальное количество запусков, что указывает на слабость выявленной связи между интересом общества и итоговым количеством запусков.

Таким образом, подтвержденная концептуальная модель с коэффициентами b представлена на рисунке 11.

Как показало исследование уравнения № 2, связь между показателями общественного интереса и финансирования существует, но ввиду её слабости и несовершенности статистических данных ее нецелесообразно использовать в итоговом варианте концептуальной модели. Таким образом, итоговая сводная система уравнений имеет следующий вид:

$$
\left\{\begin{array}{c}
y=7,92+0,32 \times 1-0,0021 \times 2+0,0051 \times 3 \\
y=11,35+0,30 x 1 \\
y=1,88-0,0014 \times 2+0,0065 \times 3
\end{array}\right.
$$

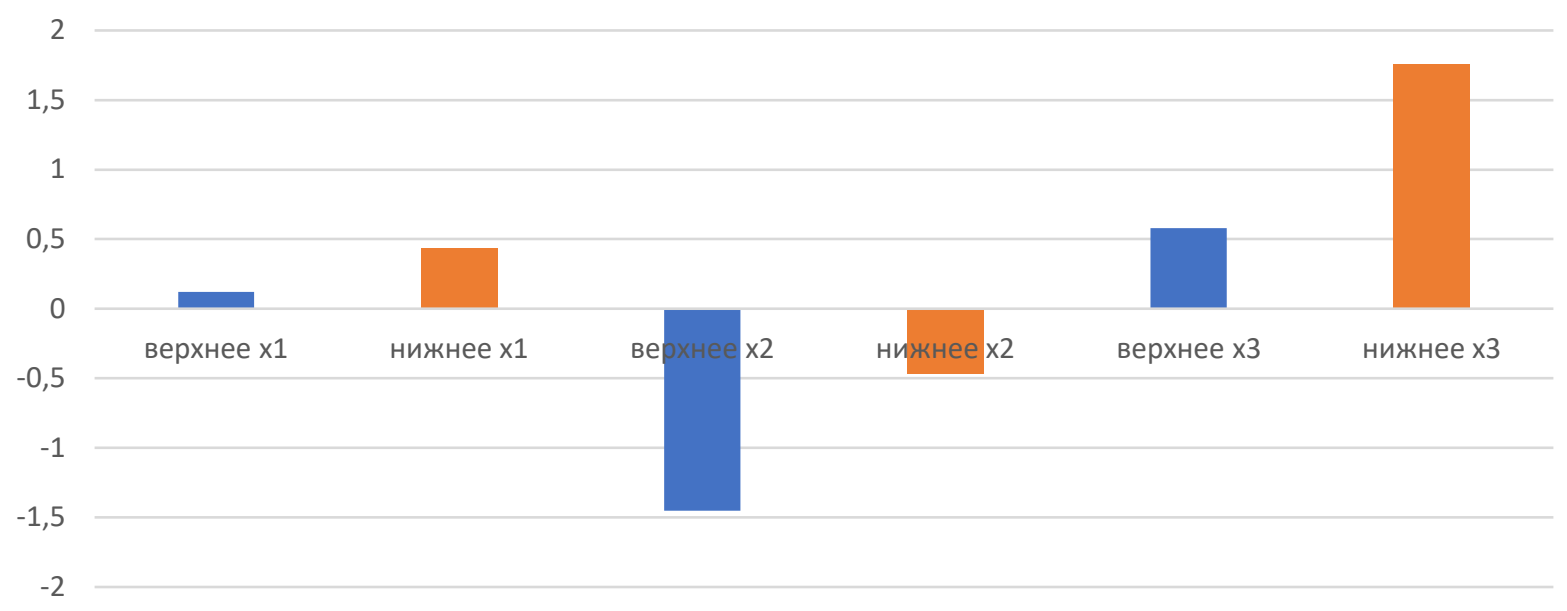

Рисунок 10. Гистограмма нижней и верхней границы доверительного интервала коэффициентов эластичности для показателей 
Кеантификаторы состояния информационной среды сферы космических исследований

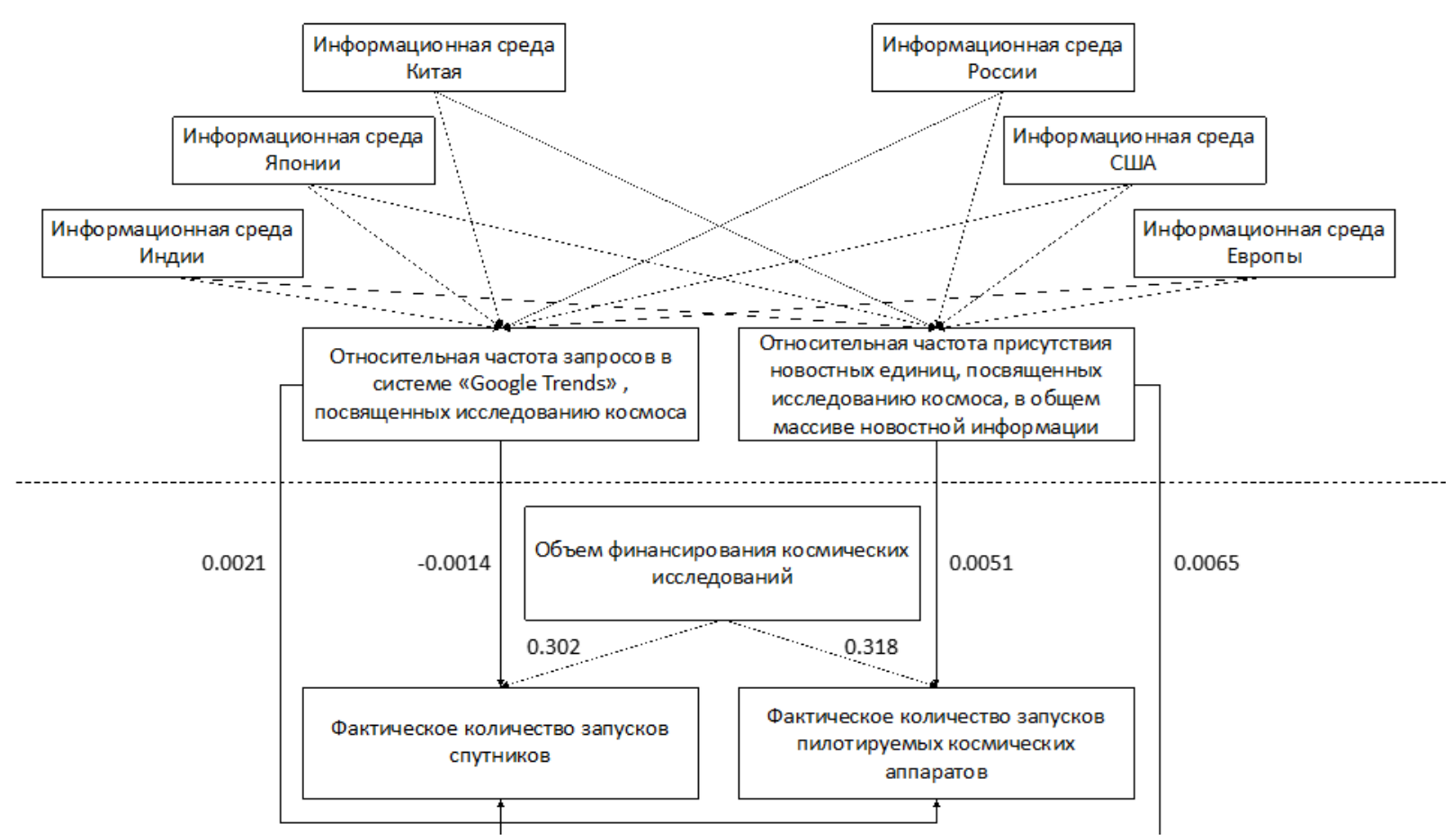

Рисунок 11. Подтверждённая концептуальная модель с коэффициентами b

Результаты анализа позволили выявить наличие взаимосвязи между рассматриваемыми показателями, но было выявлено наличие значительного воздействия объема финансирования на итоговые значения запусков. Некоторые коэффициенты, используемые при анализе, имеют отрицательное значение, что указывает на наличие отложенного эффекта. Влияние фи- нансирования на управление системой космической индустрии в целом, по нашим прогнозам, будет иметь серьезный вес следующие пять-десять лет. В дальнейшем, возможно возникновение накопительного эффекта заинтересованности общества, что позволит развивать эту сферу более активно.

\section{Библиографический список}

1. Борисова Н.М. Организационные изменения в национальной ракетно-космической отрасли как фактор развития отрасли // Научное знание современности. 2017. № 2 (2). С. 54-56.

2. Ершова А.В., Родионов Д.Г., Конников Е.А., Конникова О.А. Системный анализ привлекательности банков для представителей ВИП-сегмента потребителей в рамках цифровой информационной среды // Экономические науки. 2021. № 194. С. 60-73.

3. Катков О.Н., Штукатуров М.К. Мониторинг информационного пространства в системе подготовки специалистов-аналитиков для ракетно-космической отрасли // B книге: XLI Академические чтения по космонавтике. Сборник тезисов чтений, посвященные памяти академика С. П. Королева и других выдающихся отечественных ученых - пионеров освоения космического пространства. 2017. С. 366-367.

4. Конников Е.А., Терентьева Д. А., Конникова О.А. Анализ уровня устойчивого потребления в контексте цифровой информационной среды // Экономические науки. 2020. № 192. С. 114-125.

5. Родионов Д.Г., Конников Е.А., Конникова О.А. Методология системного анализа информационной среды // Экономические науки. 2021. № 196. С. 160-174.

6. Торопеев Д.А. Информационные технологии в ракетно-космической отрасли // В книге: Гагаринские чтения - 2018. Сборник тезисов докладов XLIV Международной молодёжной научной конференции. 2018. C. 130. 